\title{
Survey on practice of venom immunotherapy in France
}

Charles Dzviga ${ }^{1}$, Catherine Matevi², Philippe Bonniaud ${ }^{3}$, François Lavaud ${ }^{4}$, Bruno Girodet ${ }^{5}$, Joelle Birnbaum ${ }^{6}$, Claude Lambert ${ }^{7}$, the Interest Group on Insect Venom Hypersensitivity Allergy from the French Society of Allergology (SFA), French Federation of Continuous Medical Education in Allergology (ANAFORCAL)

\begin{abstract}
${ }^{1}$ Allergology Unit, University Hospital, Saint-Etienne, France ${ }^{2}$ Immunology Lab, University Hospital, Saint-Etienne, France ${ }^{3}$ Respiratory Diseases Unit, University Hospital, Dijon, France ${ }^{4}$ Respiratory Unit, University Hospital, Reims, France ${ }^{5}$ Allergology Unit, St. Luc Hospital, Lyon, France

${ }^{6}$ Allergology Unit, APHM University Hospital, Marseille, France

${ }^{7}$ Immunology Laboratory, University Hospital, Saint-Etienne, France
\end{abstract}

Submitted: 21 February 2014

Accepted: 27 May 2014

Arch Med Sci 2016; 12, $1: 150-155$

DOI: 10.5114 /aoms.2016.57591

Copyright (c) 2016 Termedia \& Banach

\section{Abstract}

Introduction: Venom immunotherapy (VIT) is the only efficient prevention for sting-induced anaphylaxis, but its application is not without risks and needs precautions and standardization. European guidelines were proposed in 2005, but recent practice surveys and more recent knowledge raise the need for an update. The aim of this study was to analyze VIT practices in France, based on previous surveys in Europe but also extended to outcome event management.

Material and methods: A paper questionnaire was sent widely to persons involved in venom treatment.

Results: Eighty-six responses could be included from physicians actively involved in VIT induction evenly distributed in France. The survey shows that VIT was engaged from grade III down to grade I reactions, starting preferentially with the ultra-rush protocol. Premedication was used by $42 \%$ only and risks induced by co-treatment with $\beta$-blockers were well known but not with angiotensin-converting enzyme inhibitors. However, side effects were very variably managed from arrest to enhancement in doses, time-delay or duration. Similarly, we observed a large discrepancy in treatment evaluation (skin tests, biology, timing and interpretation), decision making for treatment termination (when and how long to be prolonged) and post-treatment follow-up (adrenaline kit, event record) as well as procedures in case of late relapse (new induction, different doses).

Conclusions: Our study shows that most recommendations were fully or partially followed and may need reminding, but many points need to be completed or updated with new tools and knowledge acquired during the last 10 years.

Key words: immunotherapy, venom allergy, practice survey.

\section{Introduction}

Systemic reactions to hymenoptera sting can concern up to $8.9 \%$ of the population in Europe [1]. Approximately 40\% are life-threatening and gravity factors include age, cardiovascular or respiratory diseases and most of all mastocytosis. Angiotensin-converting enzyme can increase the risks of severe allergy while $\beta$-blockers can reduce its adrenaline

\author{
Corresponding author: \\ Dr Claude Lambert \\ Immunology Laboratory \\ Pole de Biologie-Pathologie \\ University Hospital \\ CHU St. Etienne \\ F 42055 St. Etienne Cedex 2 \\ France \\ Phone: +33477120513 \\ Fax: +33477120552 \\ E-mail: Claude.lambert@ \\ uni-st-etienne.fr
}


treatment efficacy $[2,3]$. Venom immunotherapy (VIT) is the only preventive treatment with $80 \%$ to $95 \%$ efficiency in terms of mortality and morbidity provided the specific venom responsible was clearly identified [1, 4]. It consists in injections of rapidly increased doses of rapidly increasing doses (induction phase) followed by periodic injections of high doses for classically 5 years and possibly longer (maintenance phase). A few protocols have been proposed for the induction phase in 15 days (classical), 3 days (rush) or even 3:30 hours (ultra-rush) [4-6]. The target dose for continuous injection should classically reach $100 \mu \mathrm{g}$, which represents approximately 2 natural doses of sting. However, medical injection of venom in allergic patients also includes some risks and requires precautions, especially in high risk patients who are also the most in need of VIT [4]. In France, VIT is usually performed by a reduced number of experts, and European guidelines were published several years ago by the EAACl working group [7]. After 10 years, it is time to evaluate the applicability of the guidelines and the needs for updating regarding recent developments since their publication. Indeed previous surveys have shown that recommendations were not always applied regarding the local health organization and daily practice constraints [8,9].

The aim of this work was to evaluate the practice in France, compared to the 2 previous studies, and identify the needs for updating of the recommendations. This is the first extensive survey performed in France on VIT.

\section{Material and methods}

The questionnaire was based on the previously published surveys in the UK and Poland [10, 11]. The Diwakar questionnaire asked questions on skin test procedures (order, dosages, venom tested), IgE dosages, basal tryptase, VIT procedures (type, doses, duration frequency, evaluation and premedication), decision making in case of discordance or side effects, and efficiency evaluation. We have added questions on IgE specificities tested including cross reactive carbohydrate determinants (CCD) and components, decision making in case of double sensitization, and late follow-up. Physicians involved in venom therapy are frequently practicing in different places and some of them only do maintenance treatment but not diagnosis or VIT decision making. In order to reach most of the concerned physicians, we sent a great excess of questionnaires (300) to any correspondents directly or indirectly involved in venom injections in 2010. The questionnaire was completed with questions on the diagnostic process that have been analyzed previously (Charles Dzviga submitted). Only responses from the physicians involved in the decision making and induction of the treatment were considered. The number of experts involved in VIT was estimated at 100-120 in France.

\section{Statistical analysis}

Data collected were analyzed using an Excel data sheet and statistical tools. Results are expressed in frequency or item chosen related to the number of responders or mean values and one standard deviation and compared using Student's or $\chi^{2}$ tests.

\section{Results}

Among 88 responses, 86 were provided by practitioners effectively involved in decision making and inducing VIT (Table I). Among them, 35 (41\%) were exclusive allergists while 39 (46\%) were pneumologists and 4 (5\%) dermatologists and 7 (8\%) pediatricians. The majority were treating from 6 to 30 new patients a year.

Our study reveals that $40 \%$ of practitioners indicated VIT at grade II or $21 \%$ even at grade I, with $39 \%$ of them taking into account the associated medical conditions that increase the risk for anaphylaxis by starting VIT at an even lower grade.

All but one practitioner executed the induction phase in a hospital environment. The ultra-rush protocol was preferentially used (60\%) except by pediatricians (30\%; Table II). Other protocols used were rush (23\%) and rarely the classical method. The rush duration protocol could vary between 2 and 3 days.

Premedication was used systematically by $42 \%$ of practitioners. It was occasionally used by $30 \%$ more in case of severe (5\%) or even local (12.5\%) reaction in previous VIT injections. It always con-

Table I. Survey on practice of venom immunotherapy in France from 86 practitioners effectively involved in decision making and practice of VIT (\% of responses) treatment induction

\begin{tabular}{|c|c|}
\hline & $N(\%)$ \\
\hline \multicolumn{2}{|l|}{ Your practice? } \\
\hline Allergology exclusively & $35(41)$ \\
\hline Pulmonology & $39(46)$ \\
\hline Dermatology & $4(5)$ \\
\hline Pediatrics & $7(8)$ \\
\hline \multicolumn{2}{|c|}{ From what stage do you usually indicate VIT? } \\
\hline Grade III & $17(27)$ \\
\hline Grade II & $25(40)$ \\
\hline Grade I & $13(21)$ \\
\hline Regional & $7(11)$ \\
\hline \multicolumn{2}{|c|}{ Do you indicate more VIT in case of } \\
\hline Medical risks & $24(39)$ \\
\hline
\end{tabular}


C. Dzviga, C. Matevi, P. Bonniaud, F. Lavaud, B. Girodet, J. Birnbaum, C. Lambert, the Interest Group on Insect Venom Hypersensitivity Allergy from the French Society of Allergology (SFA), French Federation of Continuous Medical Education in Allergology (ANAFORCAL)

sisted of an anti-histamine drug, rarely associated with corticosteroids (1 case), anti-leukotriene (1 case) or other (3 cases).

Sixty-eight (92\%) practitioners knew the added risks of $\beta$-blocker treatment and considered that it should be absolutely (46\%) or only when possible (46\%) stopped during VIT induction, 47 of them (74\%) referring to the prescriber of the treatment. On the other hand, possible added risks induced by angiotensin-converting enzyme were either ignored $(8 \%)$ or neglected $(61 \%)$ by practitioners.

In the event that a systemic reaction appeared during the induction phase, $50 \%$ of practitioners reported changing the protocol, $19 \%$, $14 \%$ and $17 \%$ in case of reaction of grade 1,2 or 3 respectively. They thus either introduced premedication as mentioned, or used smaller dose escalation in each step (71\%) or increased the delay between the steps (9.3\%). On the other hand, $6(7 \%)$ practitioners declared that they just stopped the VIT.

The maintenance phase (Table III) was in a great part practiced outside of hospital facilities, sometimes referring to another allergist

Table II. Practice of VIT induction

\begin{tabular}{|c|c|}
\hline \multicolumn{2}{|c|}{ The inducing protocol you generally use: } \\
\hline Ultra-rush & $47(60 \%)$ \\
\hline Rush & $18(23 \%)$ \\
\hline Classical & $6(8 \%)$ \\
\hline \multicolumn{2}{|l|}{ Do you use premedication? } \\
\hline Always & $35(42 \%)$ \\
\hline Occasionally & $25(30 \%)$ \\
\hline \multicolumn{2}{|c|}{ How do you consider drug induced added risk? } \\
\hline \multicolumn{2}{|c|}{ B-blockers: } \\
\hline Stop if possible bb & $34(46 \%)$ \\
\hline Absolutely stop bb & $34(46 \%)$ \\
\hline No change & $8(11 \%)$ \\
\hline \multicolumn{2}{|l|}{ ACE Inhibitor: } \\
\hline Stop & $24(32 \%)$ \\
\hline Don't know & $6(8 \%)$ \\
\hline No change & $46(61 \%)$ \\
\hline \multicolumn{2}{|c|}{$\begin{array}{l}\text { Do you change the protocol in case of systemic } \\
\text { reaction during induction phase? }\end{array}$} \\
\hline Yes & $43(62 \%)$ \\
\hline From grade I & $16(23 \%)$ \\
\hline From grade II & $12(17 \%)$ \\
\hline From grade III & $15(22 \%)$ \\
\hline \multicolumn{2}{|c|}{ What change do you choose? } \\
\hline Stop VIT & $6(7 \%)$ \\
\hline Smaller steps & $61(71 \%)$ \\
\hline Longer time delay & $8(9.3 \%)$ \\
\hline Premedication & $23(48 \%)$ \\
\hline
\end{tabular}

(18\%) or local medical practitioner including a GP or nurse (37\%). The target dose was $100 \mu \mathrm{g}$ for $99 \%$ of practitioners for wasp venom and $96.5 \%$ for honey bee venom, unless the patient was at high risk, when target doses were raised to 150 (5\% for wasp and $10 \%$ for honey bee) or $200 \mu \mathrm{g}$ in $8.5 \%$ and $15 \%$ respectively. The time delay between injections was more than 4 weeks in $34 \%$ of practitioners and was extended to 6 weeks (77\% of practitioners) after 1 year (33\%), 2 years (24.4\%) or even 3 years (31.1\%).

In case of systemic reaction during the maintenance phase, $12.5 \%$ or practitioners stopped VIT, $46.6 \%$ reduced it, while $11 \%$ increased the dose and $27.4 \%$ reduced the time delay between injections (Table IV). Furthermore, 48\% introduced anti-histamine premedication before injections and $27.5 \%$ considered that VIT was not efficient enough and an induction should be repeated. The strategy was reconsidered if reactions were of grade I (31\%), II (17\%) or higher than II (43\%).

The VIT was planned for a fixed duration of 5 years for $50 \%$ or 3 years for $9.3 \%$ of practitioners or for a variable duration, $13.3 \%$ between 3 and 5 years, $8.3 \%$ between 5 and 10 years, and $7.2 \%$ for more than 10 years (Table $\mathrm{V}$ ). Practitioners felt the treatment was well followed by patients and $54 \%$ estimated that less than 5\% stopped earlier

Table III. Practice of VIT maintenance phase (\% of responses)

\begin{tabular}{|c|c|}
\hline \multicolumn{2}{|l|}{ Maintenance phase } \\
\hline \multicolumn{2}{|l|}{ Usual target dose: } \\
\hline Wasp venom $100 \mu \mathrm{g}$ & 83 (99\%) \\
\hline Honey bee venom $100 \mu \mathrm{g}$ & $82(96.5 \%)$ \\
\hline \multicolumn{2}{|l|}{ Adjusted in patients at risk: } \\
\hline Wasp venom $150 \mu \mathrm{g}$ & $4(5 \%)$ \\
\hline $200 \mu \mathrm{g}$ & $7(9 \%)$ \\
\hline Honey bee venom $150 \mu \mathrm{g}$ & $8(10 \%)$ \\
\hline $200 \mu g$ & $12(15 \%)$ \\
\hline \multicolumn{2}{|l|}{ Initial time delay: } \\
\hline 4 weeks fixed & $46(58.2 \%)$ \\
\hline 4-5 weeks & $7(8.9 \%)$ \\
\hline 4-6 weeks & $18(22.8 \%)$ \\
\hline \multicolumn{2}{|l|}{ Increased delay after: } \\
\hline 1 year & $15(33 \%)$ \\
\hline 2 years & $11(24.4 \%)$ \\
\hline 3 years & $14(31.1 \%)$ \\
\hline$>3$ years & $5(11.1 \%)$ \\
\hline
\end{tabular}


than planned, most of the time due to slackness (40\%) more than because of side effects (20\%).

The treatment efficacy was evaluated through the local effect of injection by $25.6 \%$ or periodic skin tests (66.3\%), after 1 (64\%), 2 (15\%) or 3 (15\%) years. Biological tests were used by $65.1 \%$ of practitioners but mostly specific IgE and rarely IgG4 (5\%) or cellular tests (2\%). If efficacy was not satisfactory, VIT prolongation was frequently proposed for 1 year (46\%), 2 years $(23 \%)$ or more (26\%).

Follow-up after the termination of treatment was proposed by $60.7 \%$ of practitioners, every 1 year for $54.3 \%$ of them or 2 years for $39.1 \%$, although they estimated that this was respected by less than one third of the patients. The follow-up included skin testing (60\%) and biological tests (53\%). No procedure was proposed for long-term follow-up and systemic reaction relapses were estimated at less than $10 \%$ of the patients in the first 5 years for half of the practitioners. A new VIT could then be considered by $91.5 \%$ of the practitioners according to the clinical risk and/or after a new diagnosis test.

\section{Discussion}

Our study shows that VIT practice in France is close to the EAACI guidelines [7] as reported from Poland [9] but unlike in the UK [8]. Differences may be linked to the respective health care organizations, and indeed the British Society for Allergy and Clinical Immunology (BSACl) has recently published new guidelines that do not always comply further with the EAACI guidelines [12]. In France, allergology can be performed either exclusively or as part of another specialty, mainly dermatology, pneumology or pediatrics, which show small differences in VIT practices. Our results reflect the reality and the view of the French working group on allergy to insect sting, although it was an opinion survey and responses came from 86 out of an estimated 100-120 practitioners in France, which is similar in proportion to the two other studies.

Our survey shows that a few recommendations from the EAACI guidelines [7] need a reminder: The VIT indication should be limited to patients who experienced grade III or IV reaction unless grade II was worsened by gastro-intestinal or angioedema symptoms. Since these recommendations, more risk factors have been identified, especially mastocytosis [13-15] but also cardiovascular pathology such as uncontrolled hypertension, coronaropathy or arrhythmia, or respiratory instability as in severe chronic asthma or respiratory insufficiency [7]. Anxiety and quality of life degradation due to the risk of anaphylaxis must also be considered [7].

It was reported that $\beta$-blockers could eventually reduce the efficiency of adrenalin in case of sys-
Table IV. Management of VIT side effects (\% of responses)

\begin{tabular}{|c|c|}
\hline \multicolumn{2}{|c|}{ What to do if late systemic reaction: } \\
\hline Stop VIT & $9(12.5 \%)$ \\
\hline Reduced dose & $34(46.6 \%)$ \\
\hline Increased dose & $8(11 \%)$ \\
\hline Reduced time delay & $20(27.4 \%)$ \\
\hline Anti-histamine & $36(48 \%)$ \\
\hline New induction & $22(27.5 \%)$ \\
\hline \multicolumn{2}{|c|}{ From which grade consider change: } \\
\hline From grade I & $11(31 \%)$ \\
\hline From grade II & $6(17 \%)$ \\
\hline From grade III & $15(43 \%)$ \\
\hline
\end{tabular}

Table V. VIT termination and efficacy evaluation (\% of responses)

\begin{tabular}{|c|c|}
\hline \multicolumn{2}{|l|}{ Duration of maintenance: } \\
\hline 5 years & $42(50 \%)$ \\
\hline 3 years & $8(9.3 \%)$ \\
\hline Between 3 and 5 years & $11(13.3 \%)$ \\
\hline Between 5 and 10 years & $7(8.3 \%)$ \\
\hline More than 10 years & $6(7.2 \%)$ \\
\hline \multicolumn{2}{|l|}{ VIT efficiency evaluation: } \\
\hline Effect of injection & $22(25.6 \%)$ \\
\hline Periodic skin tests: & $57(66.3 \%)$ \\
\hline After 1 year & $28(63.6 \%)$ \\
\hline After 2 years & $11(15.9 \%)$ \\
\hline After 3 years & $11(15.9 \%)$ \\
\hline slgE dosages & $56(65.1 \%)$ \\
\hline $\operatorname{slgG4}$ & $4(5 \%)$ \\
\hline Cellular tests & $1(2 \%)$ \\
\hline \multicolumn{2}{|l|}{ Prolongation if not efficient: } \\
\hline 1 year & $16(46 \%)$ \\
\hline 2 years & $8(23 \%)$ \\
\hline$>2$ years $(26 \%)$ & $9(26 \%)$ \\
\hline \multicolumn{2}{|l|}{ Post termination of VIT } \\
\hline \multicolumn{2}{|l|}{ Follow-up after termination: } \\
\hline Yes & $51(60.7 \%)$ \\
\hline Every 1 year & $25(54.3 \%)$ \\
\hline Every 2 years & $18(39.1 \%)$ \\
\hline Every 3 years & $2(4 \%)$ \\
\hline
\end{tabular}


temic reaction due to $\mathrm{VIT}$, and it was proposed to stop the treatment before injections [2, 16]. Risk of treatment arrest (for 1 or 2 days before injections and until at least 3 maintenance injections) may exceed the added risk for VIT. Our study revealed that when it is not reasonable to stop a $\beta$-blocker, practitioners preferred proceeding VIT under $\beta$-blocker: the risk of this treatment seems lower than an sting in the country without protection. The added risk of converting enzyme inhibitors is even less clear. The drug has been suspected to increase the systemic reaction for pharmacological mechanisms, but interaction with VIT efficiency has not been evaluated $[3,10,17]$.

The induction protocol to be used was not restricted by the guidelines [7], as studies have mostly shown that they were equivalently efficient [5] and respective indications depend on the practical conditions. Indeed our data show that ultra-rush was preferred in France, for wasp VIT and in adults, while rush or even the classical protocol was more frequently used for honey bee VIT in children as it is also generally preferred in the UK [8].

Anti-histamine premedication is a good treatment of immediate hypersensitivity symptoms and reduces the symptoms of VIT side effects. Our study shows that its use should be encouraged, at least during the induction phase, since this widely used treatment has very low side effects and could even increase the VIT efficacy [11], even if the mechanism is not clarified yet.

The maintenance procedure used appeared to agree quite well with the guidelines, although the time laps between injections could be increased earlier and more progressively over the 5-year schedule. The conventional target dose for VIT maintenance $(100 \mu \mathrm{g})$ was the most frequently used in our survey. It was also admitted or acknowledged that the dose should be raised in high-risk cases, but we observed that the new dose was frequently at an intermediate level $(150 \mu \mathrm{g})$, while few studies have shown that VIT efficiency was improved with a double dose [18].

We observed a wide discrepancy in the management of possible side effects. While intuitively the approach consists in rapidly stopping the protocol, this left the patient facing a high risk for anaphylaxis without any assistance. This should be considered as a lack of efficiency and VIT intensification is then needed to significantly improve the treatment efficacy and patient safety [18]. There is no procedure proposed yet for the dose escalation. Similarly, the duration of the treatment and the risk assessment when it is finished were very heterogeneous. In case of insufficient efficiency or high risk, VIT prolongation has proven its efficiency $[19,20]$, but the conditions for this decision and schedule need homogenization. The evaluation of VIT efficiency is even more difficult and needs clarification. The risk assessment should now include mastocytosis even at the infraclinical stage [21, 22].

Post-treatment procedures: Monitoring of VIT efficiency is not clarified. Skin reactivity and specific IgE levels frequently decline but most of the time are not completely abrogated $[23,24]$. The role of ex vivo basophile activation needs to be confirmed [23, 25]. Similarly, precautions to be applied after the treatment is stopped including any drug use (anti-histaminic; adrenaline self-injection kit) are very diverse and making a late event report (new sting, any reaction, etc.) would certainly be very helpful in managing long-term efficiency.

Since this study was started, a new therapeutic tool, anti-IgE monoclonal antibody (omalizumab), has been developed, which may help in VIT tolerance and possibly efficiency [26], and its possible use should also be included in new guidelines.

In conclusion, the role of VIT should be defined in some special medical conditions such as pregnancy, chronic inflammatory diseases, auto-immunity, cancer and immunotherapy or biotherapy.

\section{Conflict of interest}

All authors are members of an academic institution and have no financial engagement in conflict with the study.

The questionnaire edition was supported by Stallergènes SA, which had no involvement either in its content or in its analysis.

\section{References}

1. Bilo MB. Anaphylaxis caused by Hymenoptera stings: from epidemiology to treatment. Allergy 2011; 66 Suppl. 95: 35-7.

2. Muller UR Haeberli G. Use of beta-blockers during immunotherapy for Hymenoptera venom allergy. J Allergy Clin Immunol 2005; 115: 606-10.

3. Lombardi C, Crivellaro M, Dama A, Senna G, Gargioni S Passalacqua $G$. Are physicians aware of the side effects of angiotensin-converting enzyme inhibitors? A questionnaire survey in different medical categories. Chest 2005; 128: 976-9.

4. Birnbaum J, Ramadour M, Magnan A, Vervloet D. Hymenoptera ultra-rush venom immunotherapy (210 min) a safety study and risk factors. Clin Exp Allergy 2003; 33: 58-64.

5. Brown SG, Wiese MD, van Eeden P, et al. Ultrarush versus semirush initiation of insect venom immunotherapy: a randomized controlled trial. J Allergy Clin Immunol 2012; 130: 162-8.

6. Golden DB. Insect sting allergy and venom immunotherapy. Ann Allergy Asthma Immunol 2006; 96: S16-21.

7. Bonifazi F, Jutel M, Bilo BM, Birnbaum J Muller U. Prevention and treatment of hymenoptera venom allergy: guidelines for clinical practice. Allergy 2005; 60: 1459-70. 
8. Diwakar L, Noorani S, Huissoon AP, Frew AJ Krishna MT. Practice of venom immunotherapy in the United King dom: a national audit and review of literature. Clin Exp Allergy 2008; 38: 1651-8.

9. Cichocka-Jarosz E, Diwakar L, Brzyski P, Tobiasz Adamczyk B, Lis G, Pietrzyk JJ. Congruence of the current practices in Hymenoptera venom allergic patients in Poland with EAACl guidelines. Arch Med Sci 2012; 7 : 832-9.

10. Caviglia AG, Passalacqua G Senna G. Risk of severe anaphylaxis for patients with Hymenoptera venom allergy: are angiotensin-receptor blockers comparable to angiotensin-converting enzyme inhibitors? J Allergy Clin Immunol 2010; 125: 1171; author reply 1171-2.

11. Muller U, Hari Y Berchtold E. Premedication with antihistamines may enhance efficacy of specific-allergen immunotherapy. J Allergy Clin Immunol 2001; 107: 81-6.

12. Krishna MT, Ewan PW, Diwakar L, et al. Diagnosis and management of hymenoptera venom allergy: British Society for Allergy and Clinical Immunology (BSACI) guidelines. Clin Exp Allergy 2011; 41: 1201-20.

13. Bonadonna P, Zanotti R Muller U. Mastocytosis and insect venom allergy. Curr Opin Allergy Clin Immunol 2010; 10: 347-53.

14. Demain JG, Minaei AA, Tracy JM. Anaphylaxis and insect allergy. Curr Opin Allergy Clin Immunol 2010; 10 318-22.

15. Rueff F, Dugas-Breit S, Przybilla B. Stinging Hymenoptera and mastocytosis. Curr Opin Allergy Clin Immuno 2009; 9: 338-42

16. Finegold I. Issues in stinging insect allergy immunotherapy: a review. Curr Opin Allergy Clin Immunol 2008; 8 : 343-7.

17. Stumpf JL, Shehab N, Patel AC. Safety of angiotensin-converting enzyme inhibitors in patients with insect venom allergies. Ann Pharmacother 2006; 40: 699-703.

18. Rueff F, Wenderoth A Przybilla B. Patients still reacting to a sting challenge while receiving conventional $\mathrm{Hy}$ menoptera venom immunotherapy are protected by increased venom doses. J Allergy Clin Immunol 2001; 108: 1027-32.

19. Goldberg A Reisman RE. Prolonged interval maintenance venom immunotherapy. Ann Allergy 1988; 61: 177-9.

20. Golden DB, Kwiterovich KA, Kagey-Sobotka A, Valentine MD, Lichtenstein LM. Discontinuing venom immu notherapy: outcome after five years. J Allergy Clin Immunol 1996; 97: 579-87.

21. Gonzalez de Olano D, Alvarez-Twose I, Esteban-Lopez MI, et al. Safety and effectiveness of immunotherapy in patients with indolent systemic mastocytosis presenting with Hymenoptera venom anaphylaxis. J Allergy Clin Immunol 2008; 121: 519-26.

22. Haeberli G, Bronnimann M, Hunziker T, Muller U. Elevated basal serum tryptase and hymenoptera venom allergy: relation to severity of sting reactions and to safety and efficacy of venom immunotherapy. Clin Exp Allergy 2003; 33: 1216-20.

23. Brown SG, Haas MA, Black JA, Parameswaran A, Woods $G M$, Heddle RJ. In vitro testing to diagnose venom aller gy and monitor immunotherapy: a placebo-controlled, crossover trial. Clin Exp Allergy 2004; 34: 792-800.

24. Carballada F, Boquete $M$, Nunez R, Lombardero $M$, de la Torre F. Follow-up of venom immunotherapy (VIT) based on conventional techniques and monitoring of immunoglobulin $\mathrm{E}$ to individual venom allergens. J Investig Allergol Clin Immunol 2011; 20: 506-13.
25. Ebo DG, Hagendorens MM, Schuerwegh AJ, et al. Flow-assisted quantification of in vitro activated basophils in the diagnosis of wasp venom allergy and follow-up of wasp venom immunotherapy. Cytometry Part B, Clinical Cytometry 2007; 72: 196-203.

26. Galera C, Soohun N, Zankar N, Caimmi S, Gallen C, Demoly P. Severe anaphylaxis to bee venom immunotherapy: efficacy of pretreatment and concurrent treatment with omalizumab. J Investig Allergol Clin Immunol 2009; 19: 225-9. 